\title{
Diverse coactivator recruitment through differential PPAR $\gamma$ nuclear receptor agonism
}

\author{
Fernando Lizcano and Diana Vargas \\ Biomedical Research Center, Universidad de La Sabana, Chía, Colombia.
}

\begin{abstract}
The PPAR $\gamma$ nuclear receptor regulates the expression of genes involved in lipid and carbohydrate metabolism, and it has protective effects in some patients with type 2 diabetes. Nevertheless, the therapeutic value of the PPAR $\gamma$ nuclear receptor protein is limited due to the secondary effects of some PPAR ligands. Because the downstream effects of PPAR $\gamma$ are determined by the binding of specific cofactors that are mediated by ligand-induced conformational changes, we evaluated the differential effects of various ligands on the binding of certain cofactors associated with PPAR $\gamma$. The ligands used were rosiglitazone for treating type 2 diabetes and telmisartan for treating arterial hypertension. Functional, phenotypic, and molecular studies were conducted on pre-adipocyte 3T3-L1 and functional studies in U2OS cells. The moderating influence of various cofactor families was evaluated using transient transfection assays. Our findings confirm that telmisartan has a partial modulating effect on PPAR $\gamma$ activity compared to rosiglitazone. The cofactors SRC1 and GRIP1 mediate the activity of telmisartan and rosiglitazone and partially determine the difference in their effects. Studying the modulating activity of these cofactors can provide interesting insights for developing new therapeutic approaches for certain metabolic diseases.
\end{abstract}

Keywords: DNA binding proteins co-activator, gene expression, transcription, telmisartan.

Received: June 26, 2012; Accepted: October 4, 2012.

\section{Introduction}

PPAR $\gamma$ (peroxisome proliferator-activated receptorgamma) is a transcription factor and a member of the nuclear receptor protein family, which regulates gene expression via binding at concrete DNA sites known as PPAR $\gamma$ response elements (PPREs). The role of PPAR $\gamma$ includes regulating carbohydrate and lipid metabolism and cellular differentiation processes (Ferre 2004; Lehrke and Lazar, 2005; Shulman and Mangelsdorf, 2005). At the structural level, PPAR $\gamma$ has several functional domains. The ligand binding domain (LBD) at the $\mathrm{C}$-terminus modulates the receptor's structural conformation, thus permitting the capture of transcriptional cofactors that assist in reactions required to induce greater transcription of specific genes (Nolte et al., 1998; Bähr et al., 2011). These cofactors may act through direct changes in the structural conformation of chromatin, as is the case with the p160 family of steroid receptors (SRC) and the p300 (E1A-associated 300-kDa binding protein)/CBP (cyclic AMP response elementbinding protein) complex (Aoyagi and Archer, 2008; Bähr et al., 2011).

Send correspondence to Fernando Lizcano. School of Medicine, Universidad de La Sabana, Puente del Común Campus Universitario. Chía, Cundinamarca, Colombia. E-mail: fernando.lizcano@ unisabana.edu.co or fernandoll@unisabana.edu.co.
PPAR $\gamma$ is a key regulator in the differentiation of adipose cells. Gene expression resulting from complete activation of PPAR $\gamma$ by certain synthetic ligands can increase insulin sensitivity (Rosenfeld and Glass, 2001; Staels and Fruchart, 2005). Notwithstanding these beneficial effects, the pharmacological potential of these ligands is limited by adverse effects, including weight gain, water retention and increased concentrations of lipoproteins (Guan et al., 2005; Lago et al., 2007; Nissen and Wolski, 2007). A partial activation of PPAR $\gamma$ remains an interesting theoretical approach if the metabolic benefits can be maintained and the secondary effects be eliminated. This could be achieved through the use of partial agonists, known as selective PPAR $\gamma$ modulators (SPPARMs) (Toyoma et al., 2011). Telmisartan, an angiotensin receptor blocker (ARB), was characterized as a potential partial modulator of PPAR $\gamma$, although data on telmisartan's effects are not entirely conclusive (Schupp et al., 2005).

In this study, we evaluate the activation of PPAR $\gamma$ in the presence of rosiglitazone and telmisartan, and further investigate PPAR $\gamma$ 's ability to incorporate specific cofactors in pre-adipocyte cells. We find that both rosiglitazone and telmisartan differentially reduce triglyceride accumulation in 3T3-L1 pre-adipocyte cells, and show that the magnitude of the effect is dependent on the presence of certain cofactors. These findings are likely 
due to a dissimilar effect of these two ligands on the PPAR $\gamma$ receptor.

\section{Materials and Methods}

\section{Plasmids}

The PPAR $\gamma$ coding sequence was cloned into the SalI site of the pSV.SPORT1 vector (Tontonoz et al., 1994). The Gal4-PPAR $\gamma$ LBD and PPRE-Luc plasmids were provided by Dr. Bruce Spiegelman of the Dana-Farber Cancer Institute, Boston, MA. DNA encoding p300 was inserted between the NotI and HindIII restriction sites in the CMVNHA vector. The cloning procedures for SRC1 (steroid receptor coactivator-1), GRIP1 (glucocorticoid receptor interacting protein 1) and PRIP (PPAR $\gamma$ interacting protein) have been described previously (Onate et al., 1995; Hong et al., 1996; Zhu et al., 2000). The VP16-SRC1 vector was constructed by inserting the polymerase chain reaction (PCR)-amplified $S R C 1$ gene fragment into the EcoRI site of the VP16 activation domain (residues 409-490), The $5 \mathrm{XUAS}$ reporter gene was inserted between the $\mathrm{BamHI}$ and HindIII restriction sites in the pT109 vector, which has a TK promoter coupled with luciferase (Takeshita et al., 1998).

\section{Transient transfection and double-hybrids}

The U2OS and 3T3-L1 cell lines were obtained from the American Tissue Culture Collection (ATCC) and grown in Dulbecco's Modified Eagle's Medium (DMEM) supplemented with $10 \%$ fetal calf serum and $1 \%$ penicillin/streptomycin at $37{ }^{\circ} \mathrm{C}$ in a $5 \% \mathrm{CO}_{2}$ atmosphere. When the cells reached $70 \%$ confluence, they were transiently transfected using a lipofectamine 2000 (Invitrogen) protocol in 12-well culture dishes. Transfections were done with $0.85 \mu \mathrm{g}$ of PPRE-Luc (reporter plasmid), $0.5 \mu \mathrm{g}$ of CMV $\beta$-Gal (control plasmid), $0.2 \mu \mathrm{g}$ of PPAR $\gamma$ and $0.1 \mu \mathrm{g}$ of each of the co-activators (p300, SRC1 GRIP1 and PRIP) added to each well. The total amount of transfected DNA was normalized using an empty pcDNA3.1 vector. After $24 \mathrm{~h}$, the medium was replaced with DMEM with $10 \%$ fetal calf serum treated with activated carbon and a resin to eliminate endogenous ligands plus either $1 \mu \mathrm{M}$ or $10 \mu \mathrm{M}$ of the ligand to be tested (rosiglitazone or telmisartan). Dimethyl sulfate $\left(\mathrm{Me}_{2} \mathrm{SO}_{4}\right)$ was used as a vehicle at a maximum concentration of $0.2 \%$. After an additional $24 \mathrm{~h}$ of incubation, the cells were washed and lysed with Triton X-100 buffer, and the activity levels of both luciferase and $\beta$-Galactosidase were quantified.

For the double-hybrid study, the above protocol was used to transfect U2OS cells with $0.85 \mu \mathrm{g}$ of the 5XUASTK-Luc reporter plasmid, $0.1 \mu \mathrm{g}$ of the Gal4 PPARgamma plasmid and $0.35 \mu \mathrm{g}$ of the VP16-SRC1 and the CMVßGalactosidase plasmid was used as an internal control. The fold-change in luciferase or $\beta$-Galactosidase expression in the cells containing the experimental vectors was calculated relative to expression in the presence of only the control expression vector pcDNA3.1 The data was expressed as the mean \pm the standard error of the mean (SEM) and represent a minimum of three independent experiments, with each data point run in triplicate for each experiment.

\section{Adipose cell differentiation assay}

Pre-adipocyte 3T3-L1 cells were maintained in DMEM medium supplemented with $10 \%$ fetal calf serum and $1 \%$ antibiotics. Differentiation was induced 48 hours after the cells reached confluence using media containing $1 \mu \mathrm{M}$ dexamethasone, $0.5 \mathrm{mM}$ 3-isobutyl-1-methylxanthine and either telmisartan $(1 \mu \mathrm{M}$ or $10 \mu \mathrm{M})$, rosiglitazone $(1 \mu \mathrm{M})$ or a combination of both ligands, as shown in the results. After $48 \mathrm{~h}$, the medium was changed, and only the ligands were added; the medium was replaced every 2 days during the 8 days of treatment. Differentiated cells were then washed and fixed with $10 \%$ formaldehyde and stained with $0.6 \%$ red oil in $60 \%$ isopropanol for $2 \mathrm{~h}$ at room temperature. For the quantification step, the stained single layer was washed extensively to remove leftover dye, and $1 \mathrm{~mL}$ of isopropyl alcohol was added to differentiate the disks. After $5 \mathrm{~min}$, the absorbance was measured by spectrophotometry at 510 nanometers.

\section{Western blot analysis}

The 3T3-L1 cells were lysed in RIPA buffer [1X PBS, $1 \%$ Nonidet P- $40,0.1 \%$ SDS and protease inhibitors (Roche)]. Samples were collected at 0, 4 and 8 days after differentiation. After centrifugation at $4000 \mathrm{~g} \mathrm{x} 4 \mathrm{~m}, 200 \mu \mathrm{g}$ of total protein was mixed with an equal volume of sample buffer, and the mixture was denatured at $95{ }^{\circ} \mathrm{C}$ for $3 \mathrm{~min}$. The proteins were separated by electrophoresis in an SDS polyacrylamide gel and then transferred to a nitrocellulose membrane. The membrane was incubated in 5\% non-fat powdered milk in $0.1 \% \mathrm{v} / \mathrm{v}$ Tween- 20 in PBS for $1 \mathrm{~h}$ at $4{ }^{\circ} \mathrm{C}$ to block nonspecific binding. The membrane was then incubated with antibodies against fatty acid-binding protein 4/adipocyte lipid-binding protein (Fabp4/aP2) (ABCAm, Cambridge MA, USA) at a 1:1000 dilution. The membrane was then washed and incubated with HRP goat anti-rabbit antibody (1:2000) (ABCAm, Cambridge MA, USA) for $1 \mathrm{~h}$. The membrane was then developed with ECL solution (Amersham Pharmacia Biotech) and visualized by autoradiography. Quantitative analysis was performed by means of densitometry analyses of western blots from three different experiments. Results are expressed as means \pm SEM.

\section{Statistical analyses}

Analysis of Variance (ANOVA) tests were performed using StatView software and differences were considered sta- 
tistically significant when $p<0.05$. In the transfection studies, co-transfections with $\beta \cdot$ galactosidase were performed so as to normalize the data and avoid dispersion of the results. $\beta \cdot$ galactosidase has a different promoter reporter vector and thus does not interfere with the results. Assays were performed in triplicate. The Student $t$-test was used for comparisons of two treatments.

\section{Results}

To measure the transcriptional activation of PPAR $\gamma$ by telmisartan, U2OS cells were transiently transfected with a plasmid encoding for PPAR $\gamma$ and a reporter vector encoding for natural PPRE. Treatment of transfected cells with telmisartan significantly increased the luciferase activity. However, large doses of telmisartan $(10 \mu \mathrm{M})$ did not result in a greater increase in PPAR $\gamma$ compared to that observed when the cells were treated with rosiglitazone. We next investigated a potential synergistic effect between the two ligands on PPAR $\gamma$ transactivation. Treatment of transfected cells with telmisartan combined with rosiglitazone significantly reduced the activation of PPAR $\gamma$ compared to the level of activation observed with rosiglitazone alone (Figure 1A).

Keeping in mind that PPAR $\gamma$ plays a fundamental role in establishing the adipocyte phenotype, we analyzed the effect of agonists on the differentiation of 3T3-L1 pre-adipocytes to see if the data were in concordance with the data obtained from the functional assays. We found that rosiglitazone induces a greater accumulation of triglycerides, thus implying a higher level of differentiation, compared to high doses of telmisartan. When telmisartan was added to the differentiation medium at doses of 1 or $10 \mu \mathrm{M}$ in the presence of rosiglitazone, a reduction in pre-adipocyte differentiation was observed (Figure 1B).

A molecular evaluation of PPAR $\gamma$ transcriptional activity was performed by measuring the production of Fabp4/aP2, (fatty acid binding protein 4) and a higher level of expression was observed when the cells were treated with rosiglitazone rather than telmisartan (Figure 2).

To determine whether the observed effects were due to a difference in the incorporation of cofactors by rosiglitazone and telmisartan, we tested whether the incorporation of various cofactors resulted in different effects on PPAR $\gamma$ activity. Co-transfection with p300 and PRIP significantly increased activation of PPAR $\gamma$ by both agonists, whereas the p160 family cofactors (SRC1 and GRIP) did not result in a significant increase in PPAR $\gamma$ activity after the addition of telmisartan (Figure 3). To evaluate the interaction between PPAR $\gamma$ and SRC1 in the presence of telmisartan and rosiglitazone, we performed a double-hybrid assay in mammalian cells. When telmisartan was tested, the luciferase activity increased approximately 9-fold compared to the basal level. When the Gal4-PPAR $\gamma$ LBD and VP16-SRC1 plasmids were co-transfected, there was a slight increase in the luciferase activity. This is in contrast
A

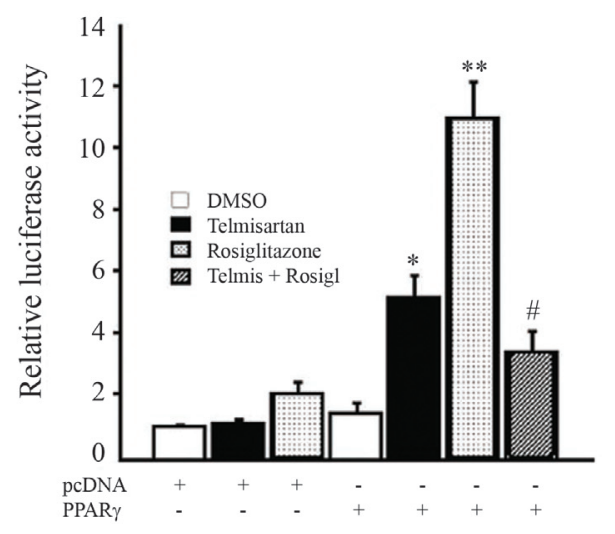

B

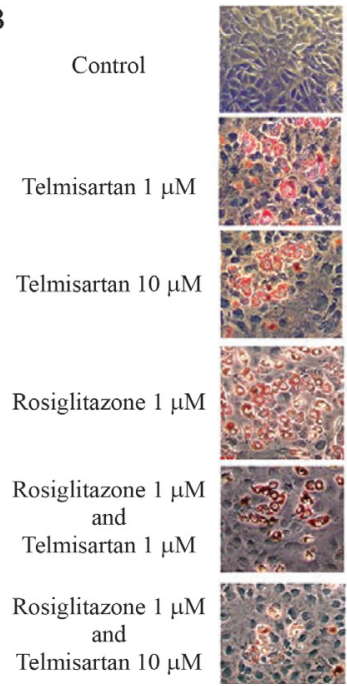

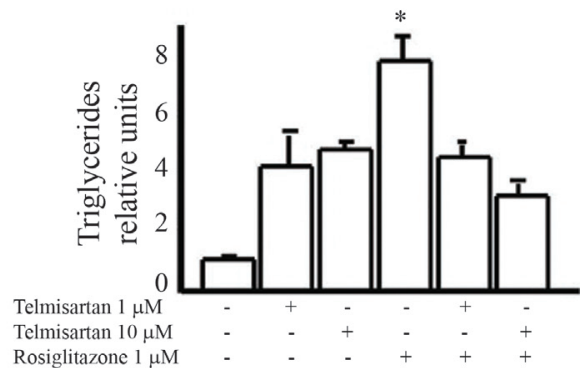

Figure 1 - Telmisartan and rosiglitazone induce PPAR $\gamma$ transcriptional activation and differentiation of adipose cells. A. U2OS cells were transiently transfected with a reporter plasmid along with luciferase PPRE-Luc and PPAR $\gamma$ vectors. The cells were then treated with $10 \mu \mathrm{M}$ telmisartan or $1 \mu \mathrm{M}$ rosiglitazone. The luciferase activity was normalized using $\beta$-Galactosidase expression, and the results were calculated from assays performed in triplicate. B. Differentiation of pre-adipocyte 3T3-L1 cells was induced with a medium containing $1 \mu \mathrm{M}$ dexamethasone, 0.5 mM 3-isobutyl-1-methylxanthine and either telmisartan $(1 \mu \mathrm{M}$ or $10 \mu \mathrm{M})$ or rosiglitazone $(1 \mu \mathrm{M})$. Triglyceride accumulation was quantified as described in the Materials and Methods section. The data are expressed as the mean $\pm \mathrm{SE}$, and represent a minimum of three independent experiments, with each data point run in triplicate for each experiment. Statistical analysis were done by Analysis of Variance (ANOVA) For A: * fold-change in luciferase expression relative to expression in the presence of only the control expression vector $\mathrm{p}<0.05$; ** fold-change in luciferase expression relative to expression in the presence of only the control expression vector $\mathrm{p}<0.01$; \# fold-change in luciferase expression relative to Rosiglitazone activation in the presence of PPAR $\gamma$ expression vector $\mathrm{p}<0.01$. For $\mathrm{B}$ : ${ }^{*} \mathrm{p}<0.05$ Rosiglitazone $v s$. Telmisartan. 

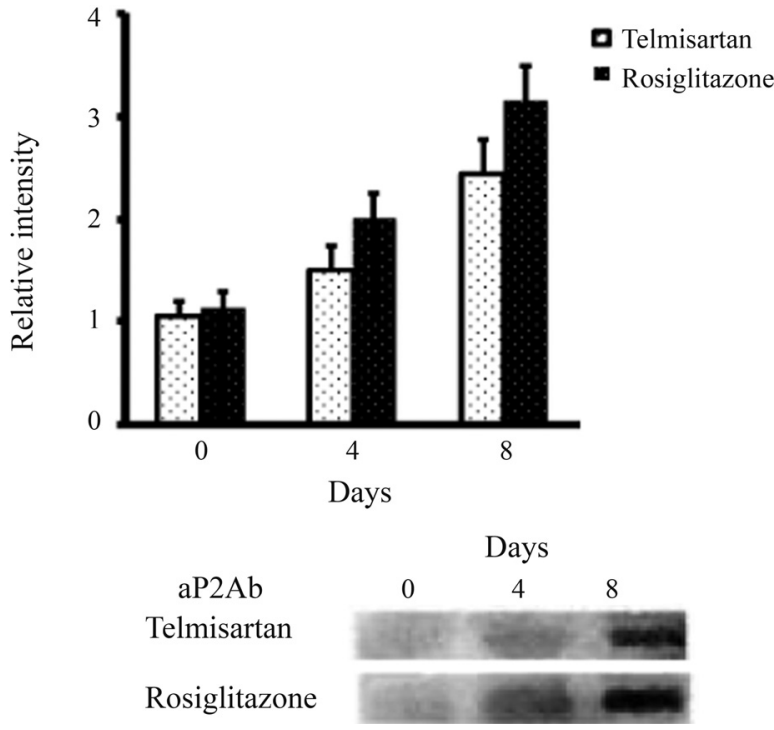

Figure 2 - Expression of aP2 after telmisartan or rosiglitazone stimulation. Pre-adipocyte 3T3-L1 cells were differentiated and treated with telmisartan or rosiglitazone. The expression levels of aP 2 were analyzed on the days shown (day 0 was defined as 48 hours after the start of differentiation) and analyzed by western blotting. Quantitative analysis was performed via densitometric analysis of the western blots in three different experiments.

to the results observed when rosiglitazone was added, which resulted in a 40-fold increase in luciferase activity; moreover, co-transfection with both SRC1 and Gal4PPAR $\gamma$ LBD led to a $100 \%$ increase in luciferase activity relative to transfection with Gal4-PPAR $\gamma$ LBD alone (Figure 4).

\section{Discussion}

PPAR $\gamma$ agonists have been good help to treat diabetes type 2 by increasing insulin sensitivity and decreasing proinflammatory adipocytokines. However, adverse effects, such as water accumulation and lightweight gain, are associated with some of these agonists (Guan et al., 2005; Lago et al., 2007). Studies of PPAR $\gamma$ expression in murine and human models have suggested that neither total agonism nor antagonism of PPAR $\gamma$ offer an optimal treatment approach for metabolic disorders (Barroso et al., 2005; Kintscher, 2012). Still, the identification of molecules that moderately activate PPAR $\gamma$ could be an important object of study.

SPPARMs are a group of ligands that interact selectively with cofactors, resulting in partial activation of PPAR $\gamma$ function and thus theoretically avoiding the adverse effects associated with total agonists (Yamauchi et al., 2001). Previous studies have shown that telmisartan, a specific and selective agonist of angiotensin II receptors, acts as a SPPARM and promotes the differentiation of adipose cells via the partial activation of PPAR $\gamma$ (Gelman et al., 2007; Destro et al., 2011). In the current study, we found

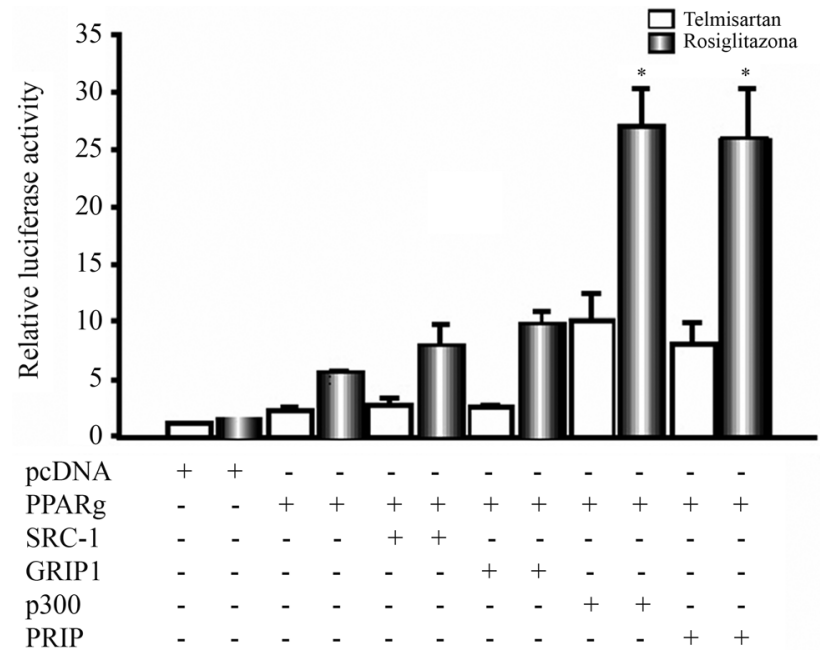

Figure 3 - PPAR $\gamma$ activation in the presence of different activating cofactors. U2OS cells were cotransfected as in Figure 1, and plasmids with different activating cofactors were cotransfected in the presence of telmisartan or rosiglitazone. Statistical evaluation was performed based on three different experiments. Test was performed Analysis of Variance (ANOVA) and differences were considered statistically significant when the mean value with standard error was $\mathrm{p} \leq 0.05$.

that telmisartan can stimulate the differentiation of 3T3-L1 pre-adipocytes both phenotypically and at the molecular level. However, the effect of telmisartan is less than the one observed after treatment with rosiglitazone (Figure 2). Our results show that the differential coupling of activating cofactors could explain the lower activation of PPAR $\gamma$ by telmisartan (Figure 4).

We furthermore found that $\mathrm{p} 300$ plays an important role in the coactivation of PPAR $\gamma$ as mediated by telmisartan and rosiglitazone. However, the p160 family of cofactors (SRC1 and GRIP1) did not induce higher activation in the presence of telmisartan (Figure 3). It is likely that these cofactors do not associate with the transcription complex in the presence of telmisartan. To further assess this hypothesis, we performed double-hybrid assays to evaluate the interaction of these proteins. We found an increase of nearly $40 \%$ in luciferase activity relative to basal levels when Gal4-PPAR $\gamma$ LBD and SRC1-VP16 were co-transfected in the presence of telmisartan. However, this same assay done in the presence of rosiglitazone showed a greater than $100 \%$ increase in luciferase activity (Figure 4).

Structural studies have shown less stabilized binding of telmisartan with Helix 12 of PPARg, which may explain the reduced recruitment of coactivators (Amano et al., 2012). Thus, we conclude that there is a decreased interaction of SRC1 with the transcriptional machinery when telmisartan is present compared to the total agonist rosiglitazone. Tagami et al., 2009, found scarce recruitment of SRC1 from PPAR $\gamma$ in mammalian two-hybrid assay with telmisartan. However, as they found an increase in PPAR $\gamma$ transactivation after GRIP1 cotransfection, one can argue 
A

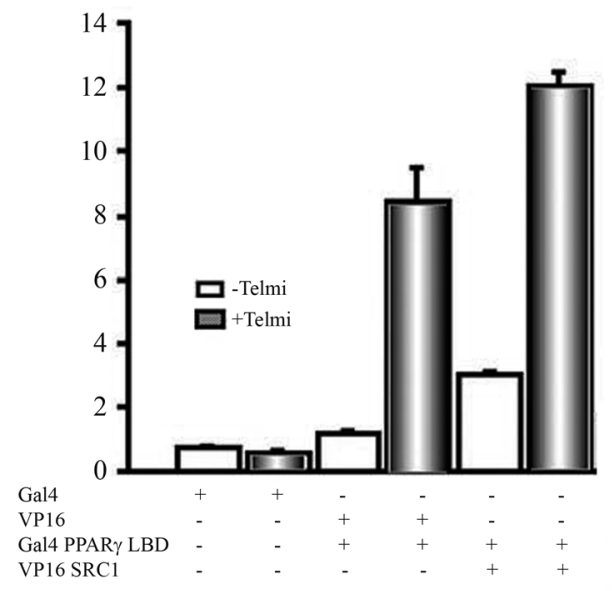

B

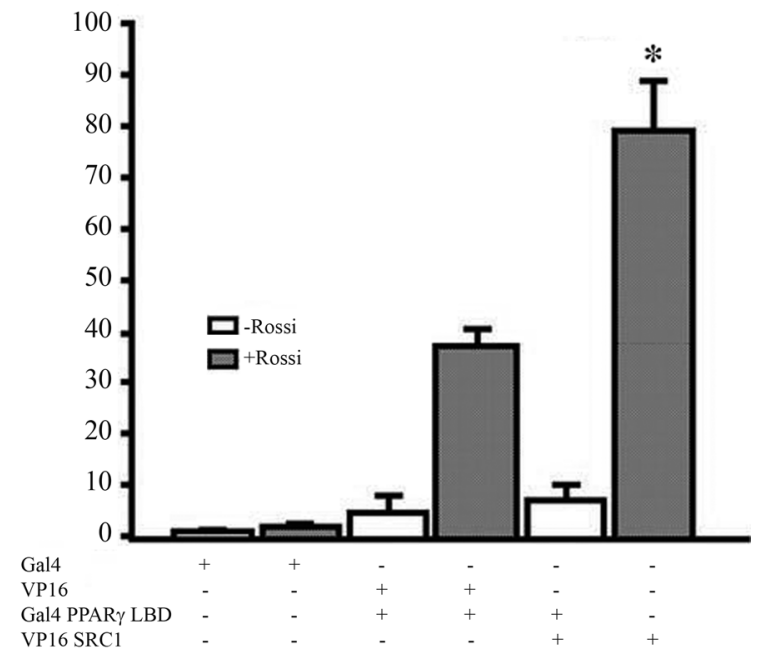

Figure 4 - Evaluation of the interaction of SRC1 cofactors with PPAR $\gamma$ in the presence of telmisartan or rosiglitazone. U2OS cells were cotransfected using the double-hybrid technique, and SRC1 interaction with PPAR $\gamma$ after treatment with telmisartan or rosiglitazone was evaluated. The data are expressed as the mean \pm SEM, and represent a minimum of three independent experiments, with each data point run in triplicate for each experiment. Test was performed Analysis of Variance (ANOVA) and differences were considered statistically significant when the mean value with standard error was $\mathrm{p} \leq$ 0.05 . As in the transfection studies are performed co-transfection with $\beta$-galactosidase, to normalize the data and avoids the dispersion of the results. $\beta$ -galactosidase has a different promoter reporter vector to thereby does not interfere with the results.

that a possibly different PPAR $\gamma$ promoter might be the cause of the differences observed. Consistent with the functional data, we found that cells treated with telmisartan showed lower accumulation of lipids compared to those treated with rosiglitazone, which had a high concentration of triglycerides.

In conclusion, this study compared the functional and molecular effects of telmisartan and rosiglitazone on the PPAR $\gamma$ nuclear receptor complex. We found that the selective recruitment of cofactors could explain the moderate activation of PPAR $\gamma$ gene expression and low accumulation of triglycerides induced by telmisartan. The characterization of substances that act as SPPARMs is of great importance, given that these compounds could result in fewer adverse effects compared to total PPAR $\gamma$ activators, while maintaining the beneficial metabolic effects associated with moderate activation of PPAR $\gamma$.

\section{Acknowledgments}

We thank Dr. Akira Takeshita, Toshi Iwasaki and Nori Kibuchi for the vector plasmids of SRC1. We also thank Ivan Martinez, Carolina Romero and Luis Celis for their technical support. This study was sponsored by La Sabana University through an internal research-funding request. The code assigned to this project by the Research Office of La Sabana University is MED-134-2010. This work was supported in part by Grant 123051929210 from Colombian Department of Science, Technology and Innovation, Colciencias. Companies that distribute telmisartan or rosiglitazone did not support this study financially.

\section{References}

Amano Y, Yamaguchi T, Ohno K, Niimi T, Orita M, Sakashita H and Takeuchi M (2012) Structural basis for telmisartanmediated partial activation of PPAR gamma. Hypertens Res 35:715-719.

Aoyagi S and Archer TK (2008) Dynamics of co-activator recruitment and chromatin modifications during nuclear receptor mediated transcription. Mol Cell Endocrinol 280:1-5.

Bähr IN, Tretter P, Krüger J, Stark RG, Schimkus J, Unger T, Kappert K, Scholze J, Parhofer KG and Kintscher U (2011) High-dose treatment with telmisartan induces monocytic peroxisome proliferator-activated receptor- $\gamma$ target genes in patients with the metabolic syndrome. Hypertension 58:725-732.

Barroso I, Gurnell M, Crowley VE, Agostini M, Schwabe JW, Soos MA, Maslen GL, Willimas TDM, Lewis H, Schafer AJ, et al. (1999) Dominant negative mutations in human PPAR gamma associated with severe insulin resistance, diabetes mellitus and hypertension. Nature 402:880-883.

Destro M, Cagnoni F, Dognini GP, Galimberti V, Taietti C, Cavalleri C and Galli E (2011) Telmisartan: Just an antihypertensive agent? A literature review. Expert Opin Pharmacother 12:2719-2735.

Ferre P (2004) The biology of PPAR receptors: Relationship with lipid metabolism and insulin sensitivity. Diabetes $53 \mathrm{Suppl}$ 1:S43-S50.

Gelman L, Feige JN and Desvergne B (2007) Molecular basis of selective PPARgamma modulation for the treatment of type 2 diabetes. Biochim Biophy Acta 1771:1094-1007.

Guan Y, Hao C, Cha DR, Rao R, Lu W, Kohan DE, Magnuson MA, Redha R, Zhang Y and Breyer MD (2005) Thiazolidinediones expand body fluid volume through PPAR $\gamma$ stimulation of ENaC-mediated renal salt absortion. Nat Med 11:861-866. 
Hong H, Kohli K, Trivedi A, Johnson D and Stallcup M (1996) GRIP1, a novel mouse protein that serves as a transcriptional coactivator in yeast for the hormone binding domains of steroid receptors. Proc Natl Acad Sci USA 93:4948-4952.

Kintscher U (2012) And in the end - Telmisartan directly binds to PPAR $\gamma$. Hypertens Res 35:704-705.

Lago R, Singh P and Nesto R (2007) Congestive heart failure and cardiovascular death in patients with prediabetes and type 2 diabetes given thiazolidinediones: A meta-analysis of randomised clinical trials. Lancet 370:1129-1136.

Lehrke M and Lazar MA (2005) The many faces of PPAR $\gamma$. Cell 123:993-999.

Nissen S and Wolski K (2007) Effect of rosiglitazone on the risk of myocardial infarction and death from cardiovascular causes. N Engl J Med 356:2457-2471.

Nolte RT, Wisley GB, Westin S, Cobb JE, Lambert MH, Kurokawa R, Rosenfeld MG, Wilson TM, Christopher KG and Milburn MV (1998) Ligand binding and co-activator assembly of the peroxisome proliferators receptor-gamma. Nature 395:137-143.

Onate S, Tsai S, Tsai M and O’Malley B (1995) Sequence and characterization of a coactivator for the steroid hormone receptor superfamily. Science 270:1354-1357.

Rosenfeld M and Glass CK (2001) Coregulator codes of transcriptional regulation by nuclear receptors. J Biol Chem 40:36865-36868.

Schupp M, Clemenz M, Gineste R, Witt H, Janke J, Helleboid S, Hennuyer N, Ruiz P, Unger T, Staels B, et al. (2005) Molecular characterization of new selective peroxisome proliferator-activated receptor gamma modulators with angiotensin receptor blocking activity. Diabetes 54:3442-3452.

Shulman AI and Mangelsdorf DJ (2005) Retinoid X receptor heterodimers in the metabolic syndrome. N Engl J Med 353:604-615.
Staels B and Fruchart JC (2005) Therapeutic roles of peroxisome proliferator activated receptor agonists. Diabetes 54:24602470.

Tagami T, Yamamoto H, Moriyama K, Sawai K, Usui T, Shimatsu A and Naruse M (2009) A selective peroxisome proliferator-activated receptor-gamma modulator, telmisartan, binds to the receptor in a different fashion from thiazolidinediones. Endocrinology 150:862-870.

Takeshita A, Yen P, Ikeda M, Cardona G, Liu Y, Koibuchi N, Norwitz ER and Chin WW (1998) Thyroid hormone response elements differentially modulate the interactions of thyroid hormone receptors with two receptor binding domains in the steroid receptor coactivator-1. J Biol Chem 273:21554-21562.

Tontonoz P, Hu E, Graves RA, Budavari AI and Spiegelman BM (1994) mPPAR gamma 2: Tissue-specific regulator of an adipocyte enhancer. Genes Dev 8:1224-1234.

Toyama K, Nakamura T, Kataoka K, Yasuda O, Fukuda M, Tokutomi Y, Dong YF, Ogawa H and Kim-Mitsuyama S (2011) Telmisartan protects against diabetic vascular complications in a mouse model of obesity and type 2 diabetes, partially through peroxisome proliferator activated recep tor- $\gamma$-dependent activity. Biochem Biophys Res Commun 410:508-513.

Yamauchi T, Kamon J, Waki H, Murakami K, Matojima K, Komeda K, Ide T, Kubota N, Terauchi Y, Tobe K, et al. (2001) The mechanism by which both hetetozygous peroxisome proliferator-activated receptor gamma (PPAR gamma) deficiency and PPARgamma agonist improve insulin resistance. J Biol Chem 276:41245-41254.

Zhu Y, Kan L, Qi C, Kanwar Y, Yeldandi A, Rao M and Reddy J (2000) Isolation and characterization of peroxisome proliferator-activated receptor (PPAR) interacting protein (PRIP) as a co-activator for PPAR. J Biol Chem 275:13510-13516.

Associate Editor: Carlos F.M. Menck

License information: This is an open-access article distributed under the terms of the Creative Commons Attribution License, which permits unrestricted use, distribution, and reproduction in any medium, provided the original work is properly cited. 